\title{
44162 - DEPTH OF ANESTHESIA, THE ENDOCRINE-METABOLIC STRESS RESPONSE AND THE POSTOPERATIVE OPIOID REQUIREMENTS
}

\author{
Gabriele Baldini, McGill University, Montreal, QC, Canada; \\ Hema Bagry, McGill University; \\ Franco Carli, McGill University;
}

INTRODUCTION: Monitoring depth of anesthesia has been shown to influence the consumption of anesthetics. Recently it has been reported that, with deep levels of anesthesia achieved with either propofol or isoflurane and opioids, postoperative consumption of morphine and pain intensity was reduced.[1,2] It is not clear whether the depth of anesthesia modifies pain intensity by influencing the endocrine-metabolic stress response. The purpose of this study was to assess the influence of two different concentrations of desflurane on perioperative plasma cortisol and postoperative morphine consumption.

METHODS: Local IRB approval was obtained for this study. The study was prospective and observer blinded, and included 20 women scheduled for elective total abdominal hysterectomy. They were randomly divided in two groups of 10 patients each, a Deep group (D) and a Light group (L). General anesthesia was induced in both groups with propofol $3 \mathrm{mg} / \mathrm{kg}$, fentanyl 0.3 $\mathrm{mcg} / \mathrm{kg}$ and rocuronium $0.8 \mathrm{mg} / \mathrm{kg}$. Desflurane was administered at two different concentrations according to Bispectral Index monitoring (BIS) Deep, 25 and Light, 50. Postoperative pain relief was achieved with patient controlled analgesia (PCA) with i.v. morphine. Blood samples were taken before, during and after surgery for the measurement of plasma cortisol, glucose and lactate. Postoperative pain visual analog scale (VAS) and morphine consumption were recorded at regular intervals for $24 \mathrm{~h}$.

RESULTS: Mean end-tidal concentrations of desflurane in the D and L groups were $9 \%$ and $4 \%$ respectively. The concentration of plasma cortisol, glucose and lactate increased with surgery in both groups, and remained elevated with no difference between the two groups. Postoperative VAS and morphine consumption were similar in both groups.

T 1- 24 = hours from end of surgery

\begin{tabular}{|c|c|c|c|c|c|c|c|}
\hline Variable & T1 & T2 & T3 & T4 & T8 & T12 & T24 \\
\hline $\begin{array}{c}\text { Morphine } \\
\text { Used }(m g)\end{array}$ & & & & & & & \\
Light & $15.3 \pm 6.2$ & $20.7 \pm 7.9$ & $25.5 \pm 9.1$ & $29.0 \pm 10.3$ & $34.0 \pm 13.1$ & $37.5 \pm 17.7$ & $45.1 \pm 24.9$ \\
Deep & $12.5 \pm 7.9$ & $21.7 \pm 7.1$ & $26.0 \pm 8.1$ & $28.5 \pm 8.7$ & $33.1 \pm 9.2$ & $36.3 \pm 10.4$ & $45.7 \pm 11.5$ \\
\hline
\end{tabular}

DISCUSSION: The results indicate that there is no relationship between the depth of intraoperative anesthesia achieved with desflurane and the extent of the endocrine-metabolic stress response or postoperative analgesic requirements.

REFERENCES:

1.Acta Anaesthesiol Scand 2003;47:804-8.

2.Acta Anaesthesiol Scand 2005;49:293-6 